\title{
AUTOCLAVE DESIGN FOR HIGH PRESSURE-HIGH TEMPERATURE CORROSION STUDIES
}

\author{
B. A. Lasebikan, A. R. Akisanya ${ }^{1}$ and W.F. Deans \\ School of Engineering, \\ University of Aberdeen, \\ Aberdeen AB24 3UE, U.K.
}

\begin{abstract}
Purpose: Many new discoveries of oil and gas field are in high pressure - high temperature (HPHT) environment. The development of such fields requires appropriate selection of materials that are able to withstand not just the service loads but also corrosive production fluids in the HPHT environment. There is a need for test facilities that can be used to assess the corrosion behaviour of suitable materials in typical HPHT environment.
\end{abstract}

Design/Methodology/Approach: The exposure of material samples to elevated pressure and temperature is usually done using an autoclave. The suitability of an existing autoclave for HPHT corrosion studies is provided together with suggestions on necessary design modifications. An alternative design of the autoclave is proposed based on functionality requirements and lifecycle cost assessment.

Findings: The existing autoclave was unsuitable for HPHT corrosion tests and modifications were very expensive to implement and/or not fool proof. A new autoclave was designed, manufactured, tested and successfully used to study the effect of aqueous solution on the corrosion of a pipe subject to combination of axial tension, internal pressure and elevated temperature.

\footnotetext{
${ }^{1}$ Corresponding Author. Email: a.r.akisanya@abdn.ac.uk. Fax: +44 1224272497
} 
Research Implications/limitations: The maximum design pressure of $15 \mathrm{MPa}$ is more than sufficient for high pressure corrosion studies in aqueous solution where partial pressure of the dissolved gas is one of the main controlling parameters. However, the design pressure is only suitable for corrosion studies in seawater environment up to $1500 \mathrm{~m}$ of water depth.

Originality: A new design of autoclave together with all the necessary piping, assembly and control system is proposed for high pressure - high temperature corrosion studies. The autoclave can be used as standalone or integrated with mechanical testing machine and thus enables corrosion studies under a wide range of loading.

\section{Keywords:}

High pressure and elevated temperature; autoclave; design specification and functionality; corrosion tests; whole life costing; systems design; design, implementing, managing and practicing innovation; experimental mechanics; material, component and systems performance

\section{Introduction}

Autoclave are designed for and used by various industry sectors, for example, healthcare (Bahadori, 1976; Oyawale and Olaoye, 2007), aerospace (Upadhya et al. 2011), energy (Jenner, 1986) and oil and gas (McNamee and Conrad, 2011). The trend towards safe usage of the least-expensive materials of construction in the oil and gas industry, particularly for high pressure and high temperature (HPHT) environments, requires progressively higher accuracy in modelling and preparing test environments formulated for the appropriate service conditions. Most corrosion studies are usually carried out at ambient temperature and atmospheric pressure. However, many new discoveries of oil and gas reserves are in high pressure ( $>69$ $\mathrm{MPa})$ and high temperature $\left(>149^{\circ} \mathrm{C}\right)$. The mechanical and corrosion behaviour of many of the materials used in drilling and completions are affected not just by the 
downhole fluids but also by the pressure and temperature. It is therefore important to be able to assess the corrosion behaviour of these materials in such environment.

The exposure of material samples to elevated pressure and temperature is usually done using an autoclave, which is a pressure vessel with facility for heating. For corrosion studies at HPHT, the autoclave must, in addition to the pressure and temperature, allow the exposure of the sample(s) to the corrosive fluid/environment. There is currently no off-the-shelf autoclave for corrosion studies at HPHT in a wide range of corrosion fluid. The design of such an autoclave system is complicated by the need to allow for (i) tests in aerated environment where oxygen removal is not necessary or in deaerated (i.e. oxygen free) environment, (ii) the use of sweet or sour fluid with minimal effect on the structural integrity of the autoclave, (iii) accurate and reliable in-situ measurement of corrosion parameters, and (iv) integration with mechanical testing machine to enable corrosion studies under combined pressure, temperature and externally applied tension/compression.

Commercial and existing custom made autoclaves lack the design features for HPHT corrosion studies under combined loading. Bahadori (1976) presented the design solution for a standalone solar-based autoclave for the sterilisation of medical equipment. The design of a standalone autoclave suitable for HPHT hydrometallurgical reaction studies is given by Jenner (1986) and Holliday and Honey (1993). An inner sleeve of titanium steel and outer low alloy steel shell was used by Jenner (1976) while Holliday and Honey (1993) used a Pyrex glass inner liner and a stainless steel outer shell. The glass inner liner is attacked by water at temperature above $100{ }^{\circ} \mathrm{C}$ leading to significant wear and a compromise of the integrity of the autoclave. Further, the design by Jenner (1976) and Holliday and Honey (1993) can not be used for combined pressure and external tensile load corrosion studies.

The design of an autoclave for high pressure - high temperature corrosion studies under combined loading of the test sample is presented in this paper. The steps taken by the authors to overcome the above challenges in the design of a specialised 
autoclave for corrosion studies are described. First, the initial design options are presented with justifications for the rejection or selection of a particular option. This is followed by the detailed analysis of the chosen design option and a discussion of corrosion tests used to verify the reliability of the design. The novel aspects of the proposed design are the simplicity of the control system, portability, and capability for combined high pressure, high temperature and externally applied tension or compression load via a mechanical testing machine.

\section{Design requirements}

The required autoclave for corrosion studies must satisfy the following requirements:

(i) A pressure capacity of at least $10 \mathrm{MPa}$ (100 bar). It would be desirable to be able to accurately control the rate of pressurisation and depressurisation, but this is secondary.

(ii) Provide a temperature of up to $200^{\circ} \mathrm{C}$. It would be desirable to be able to accurately control the rate of heating and cooling, but this is secondary.

(iii) Able to accommodate a range of corrosive fluid (e.g. brine with ammonium bisulphite (ABS), $\mathrm{H}_{2} \mathrm{~S}$ and/or $\mathrm{CO}_{2}$ ) and pressurised fluid that is not damaging to the autoclave materials and components.

(iv) Have a large enough chamber to accommodate a wide range of specimen configuration (e.g. C-ring, four-point bend, uniaxial tensile, and mini pipe) for assessing corrosion under a range loading conditions.

(v) Suitable for the application of external mechanical load (static or dynamic) to the test specimen in addition to the pressure and temperature in the autoclave.

(vi) Suitable for corrosion studies of pipe samples subject to a combination of internal pressure, external pressure, axial load, and elevated temperature.

(vii) Have provisions for instrumentation that enable accurate in-situ data collection, including electrochemical measurements. 
Requirement (iv) above was to enable the study of corrosion at HPHT under a range of loading conditions. The C-ring specimen configuration is normally used to examine the corrosion behaviour in the presence of the hoop stress induced on pipes by internal pressure while four-point bend and tensile specimens are used to study the effects of longitudinal stresses induced by the axial or longitudinal stress (Nice, Kopliku and Scoppio, 1997). A mini pipe however can be used to simulate the combined effect of hoop stress and axial stress simultaneously.

\subsection{The initial design option}

The initial approach was to use an existing HPHT test facility within the School of Engineering to conduct the HPHT corrosion studies for the specimen configurations listed in requirement (iv) above. This required a careful assessment and health and safety review of the suitability of the facility for the proposed tests.

The HPHT test equipment in the School of Engineering (see Figure 1) has a number of unique facilities:

- Three HPHT chambers, capable of temperatures up to $250^{\circ} \mathrm{C}$ and pressures up to $200 \mathrm{MPa}$; thus satisfying design requirements (i) and (ii).

- Two of these chambers are equipped with external mechanical $100 \mathrm{kN}$ capacity test machines to load specimens inside the chamber through a dynamic seal (Figure 1); this satisfies requirement (v).

- Low strain rate dynamic loading, frequency up to $1 \mathrm{~Hz}$ and fluctuating load limited to $50 \mathrm{kN}$.

- Silicon oil is used as the pressuring medium. Testing in various environments is possible but must be isolated from the silicon oil.

- There are provisions for high pressure lead-through for instrumentation and in-situ data collection; this satisfies design requirement (vii). 


\subsection{Modifications to initial design option}

Due to the likelihood of crack development if corrosive fluid was used directly in the HPHT chamber and its piping system, there were only two options: (i) lining or coating the inner surface of the HPHT chamber and carrying out major modification of the piping system, or (ii) to design a smaller autoclave which will contain the corrosive fluid and the test sample, and can then be placed in the chamber of the existing HPHT for the application of pressure, temperature and external mechanical load.

The coating option was discounted because of concerns about integrity of the coating at high pressure and temperature, maintenance and the potential for hydrogen permeation. The lining of the HPHT chamber was also discounted because it was too expensive and not fool proof (Holliday and Honey, 1993), and the heating medium has to be excluded from the test sample. In addition plumbing modification (valves, pipes etc.) will be required at significant cost and not guaranteed to succeed.

The option of using an additional autoclave was therefore pursued because the HPHT chamber heating is based on the circulation of silicon oil around the chamber. The second (and smaller) autoclave will serve two functions, i.e. contain test piece in a corrosive environment and isolate the silicone oil heating medium from the test environment; thus satisfying design requirement (iii). However, in order to ensure that the smaller autoclave satisfies design requirement (iv) the load requirements and the size of the entire candidate test specimens ( $\mathrm{C}$ ring, 4-point bend, tensile and mini pipe) must be determined. It is important that the smaller autoclave is large enough to accommodate each of all the test specimens while at the same time small enough to enable its deployment inside the chamber of existing HPHT test facility. The internal dimensions of the chamber for the existing HPHT vessel are shown in Figure 2. The new smaller autoclave must freely fit into the internal volume of the chamber.

The use of four different types of specimen configurations were considered (see Figure 3): (i) C-ring for the study of material corrosion when subject to simulated 
hoop stress, (ii) the four-point bend and the uniaxial tensile specimen to study the effect of axial loads, and (iii) a mini pipe for the study of combined internal pressure and axial load. The uniaxial tensile and mini pipe specimens were chosen for this investigation as they can be used to examine the effects of hoop stress through internal pressurisation, axial tension and also the combined effect of hoop and axial stresses. The rest of this paper will therefore focus on the design of the tensile specimens, mini pipe and associated fixtures for corrosion studies in a HPHT environment.

\subsubsection{Mini pipe}

The mini pipes were machined from the longitudinal direction of a parent pipe which has 7" diameter and $14 \mathrm{~mm}$ wall thickness. The tensile specimen and mini pipes were 50 and $60 \mathrm{~mm}$ in length, respectively. Uniaxial tensile and internal pressurisation of the mini pipes was to be carried out using the dynamic HPHT chamber (Figure 1 and Figure 2). In order to apply axial tension and internal pressure an initial load assembly (Figure 4) was configured to ensure the test was feasible due to the space restriction within the HPHT test chamber. All the fixtures required to facilitate testing were fabricated from Inconel alloy C276 to the dimensions provided in Figure 5.

A mini valve, made from aluminium alloy, was sought and purchased (from Eddie Harrison Limited ${ }^{2}$ ) and fittings made to ensure the pipe could be internally pressurised and then sealed before axial tension was applied. The mini valve was to be enclosed in the connector identified as Detail 3 to prevent contact with the corrosive fluid (see Figure 4b and Figure 5). The mini pipe and fixtures assembly shown in Figure $4 \mathrm{~b}$ were to be loaded in the new autoclave which was to be placed inside the chamber of the existing HPHT test facility.

After the evaluation of the load requirements of the mini pipe samples and fabrication of the required fixtures it was now possible to establish the dimensions of the second

\footnotetext{
2 Eddie Harrison Ltd, 69 Forest Park, Stonehaven, AB39 2GF, U.K. (www.eddieharrisonlimited.co.uk).
} 
(smaller) autoclave for corrosion tests in the HPHT chamber. As a result of extensive investigation the Bellows design (to be manufactured by John Cardwell Limited ${ }^{3}$ ) and the use of miniature valves (to be supplied by Beswick Engineering4) were considered a viable solution for the second autoclave. The material of choice for the Bellows was Inconel alloy C276. The Bellows had to fit inside the HPHT chamber and also allow enough space for the oil to circulate and provide heat to the required temperature. The Bellows were to be fabricated to the dimensions given in Figure 6. The Bellows configuration was chosen for better heat distribution. The valves were to be bought from Beswick Engineering and then supplied to John Cardwell Ltd. for fitting to the Bellows as illustrated in Figure 6.

The proposed test required a combination of axial load to be provided by the dynamic vessel in the existing HPHT chamber and application of internal pressure to the mini pipe using an external nitrogen gas cylinder. Thereafter, mini pipe and the fixtures in the Bellows all had to be enclosed. The mini valve design pressure of 20.7 MPa (207 bar) was not high enough and the pressuring medium required additional infrastructure (e.g. pumps, gas cabinet, tubing, regulator and valves) at significant cost to ensure the maximum pressure of $48.3 \mathrm{MPa}$ ( $483 \mathrm{bar}$ ) expected in the test could be achieved. The mini valve option was therefore considered not feasible because of the requirement to achieve and maintain high pressures during the testing of the mini pipes. All the mini valve issues associated with the use of a separate autoclave (i.e., the Bellows) inside the HPHT test chamber were not surmountable because of the requirement for high test pressures which were greater than the pressure capacity of some of the fixtures. In addition the cost of the Bellows and supporting infrastructure was significant and not guaranteed to succeed long term, thus the Bellows option and the use of existing HPHT facility for HPHT corrosion studies was shelved. Although this initial option was dropped, as a design process it provided opportunity for conceptualization and evaluation of ideas.

\footnotetext{
${ }^{3}$ John Cardwell Limited, Stockport SK3 0SB, UK; (www.cardwell-limited.co.uk).

${ }^{4}$ Beswick Engineering Company Ltd., 21 Cowley Road, Blackpool FY4 4NE, UK; (www.beswick.com).
} 


\section{Autoclave design - Final solution}

Due to the extensive modifications and cost required to enable to the use of existing HPHT facility, it was decided to design a separate standalone specialised autoclave with all the specific requirements of the proposed experimental work as listed in section 2. The vessel and supporting structure had to be configured for corrosion tests using available Instron mechanical testing machines (Instron 1185 \& 4483) for load application. Autoclave design is determined by various parameters. However, the most important design parameters are temperature, pressure, required internal volume, vessel material and number of needed ports for instruments, loading devices and other fittings.

To ensure tests could be carried out at HPHT conditions the maximum working temperature and pressure specified was $220{ }^{\circ} \mathrm{C}$ and $15 \mathrm{MPa}$ (150 bar) respectively. Consistency in corrosion results requires negligible change in the solution chemistry during corrosion test. Further, the volume of the test solution must be sufficiently large to minimise contamination of the solution by corrosion products and to enable corrosion test samples of a reasonable size to be used (NACE Standard, 1996; Francis and Carter, 1976). Thus the test cell should be able to contain a test solution volume (i.e. internal volume of the autoclave minus the volume occupied by the specimen and all the fixtures within the autoclave) of minimum $100 \mathrm{ml}$ per square centimetre of test area (ASTM G150, 2004). The test samples are mainly uniaxial tensile specimens or mini pipes (Figure $3 c \& d$ ). Based on the dimensions of the test samples, the minimum to maximum solution volume was $520 \mathrm{ml}$ and $630 \mathrm{ml}$ for the tensile sample or mini pipe respectively. Thus a vessel internal volume of 1.4 litres was specified to ensure minimum required volume is maintained when the furniture volume is considered. Here, the term furniture refers to specimen and all other loading fixtures.

The main factors that influenced the material selection were strength, corrosion resistance, resistance to hydrogen attack and fracture toughness. Due to cost constraints the initial approach was to fabricate the vessel from carbon steel with a PTFE inner liner. However, this approach was discarded due to concerns about gas 
permeation of the liner (PTFE), functionality and ease of maintenance of the vessel. Therefore though much more expensive (compared to carbon steel) a corrosion resistant alloy material, Inconel alloy C276, was chosen.

Based on the required length of test samples and fixtures and the volume of solution, the internal diameter of the vessel was estimated to be 5" (127 mm) and internal length 4" (102 mm). The mechanical design of the pressure vessel was carried out in accordance with the requirements contained in the ASME Boiler and Pressure Vessel Code, Section VIII, Div 1, static conditions (ASME, 2007), i.e. minimum requirements for safe construction and operation. The vessel was designed to have bolted end caps with metallic seal. Centrally positioned through holes in the end caps had O-ring seals incorporated in them to enable the use of pull rods for axial loading without any leakage of the corrosion fluid from the chamber of the vessel. The seals were machined from PTFE and PEEK (Poly Ether Ether Ketone) and made to be replaceable.

The wall thickness and removal lid of the vessel were based on the ASME code equation for a cylindrical shell. The required wall thickness for an internal pressurised circular cylindrical shell was calculated using (ASME, 2007)

$$
t_{p}=\frac{P r}{S E_{1}-0.6 P}
$$

where $t_{p}$ is the required wall thickness, in inches (minus corrosion allowance); $P$ is the internal pressure, psig; $r$ is the internal radius of the vessel in inches; $S$ is the allowable stress of the vessel material at test temperature in psi; and $E_{1}$ is the longitudinal weld joint efficiency. The design pressure for the vessel was $P=15 \mathrm{MPa}$ (2165 psig), the yield strength of Inconel C276 at the expected maximum test temperature of $220^{\circ} \mathrm{C}$ was $15.5 \mathrm{ksi}(100 \mathrm{MPa})$, and $E_{1}=1$ as there was no welding in the vessel. Using these values the nominal wall thickness of the vessel was estimated to be 1 " $(25.4 \mathrm{~mm})$, which include allowance for corrosion throughout the life of the vessel as recommended by ASME Standard (ASME, 2007). 
Heating of the solution was by electric induction of the vessel wall. With the specified requirements of liquid volume, design pressure, temperature, vessel diameter and wall thickness all specified by the authors, the manufacturer, Cortest Inc. 5 , was charged with the responsibility of deciding the size of the end cap and the associated number and size of bolts; the final design of the end cap and seal arrangements are provided as a guide in Figure 7. The end caps, which were also fabricated from Inconel alloy C276, contained holes for connections to drains, inlet and outlet test gases supplies; heat exchanger (condenser); and access fittings for electrodes and other instruments etc.

The autoclave with the design requirements and parameter described above was manufactured, complete with a touch screen control panel, by Cortest Inc. A schematic of the configuration of the vessel, fittings and accessories is provided in Figures 8 and 9 (courtesy of Cortest Inc.). One of the pull rods was bored to allow for the internal pressurisation of the mini pipe (see Figure 8).

The final design parameter of the vessel, fittings and accessories are as follows. Internal diameter, $127 \mathrm{~mm}$; Wall thickness, $38 \mathrm{~mm}$; end cap thickness, $36 \mathrm{~mm} ; 1.44$ litre internal volume capacity; $18 \mathrm{~mm}$ PTFE compression seals top and bottom; one pair of $18 \mathrm{~mm}$ adaptors for $8 \mathrm{~mm}$ threaded tensile samples (both with clevis holes); one pair of $18 \mathrm{~mm}$ adapters for $10 \mathrm{~mm}$ threaded mini pipe, one with $6.35 \mathrm{~mm}$ internal diameter (to facilitate application of internal pressure to mini pipe) and one with clevis hole; 8 top ports (pressure transducer, thermocouple, condenser connection, pressure relief valve, 3 electrodes) and 4 bottom ports (drain, gas in $\mathrm{x} 2$ and gas out); and $220 \mathrm{~V}$ heating and insulating jacket to minimise heat loss but also provide personnel protection.

The instrumentation provided with the vessel was: temperature sensor, proportional integral derivative (PID) temperature controller, thermocouple, and pressure transducer. These components work in harmony with one another to achieve smooth

${ }^{5}$ Cortest Inc., 3653 Lost Nation Road, Willoughby, Ohio 44094. (www.cortest.com). 
approach to a control at a set point. PID control is the most common modern method for process control.

A purpose-built frame with an in-built jack that facilitated upward or downward movement of the vessel during mounting on testing machine was also designed and manufactured (Figure 10). After fabrication, the vessel was proof tested to a pressure of 22. $5 \mathrm{MPa}$, i.e. 1.5 times design pressure; the vessel passed the proof test and no leaks were observed in any of the fittings or connections. As part of the quality control chemical analysis of the vessel material (Inconel Alloy C276) was also required to ensure vessel material was per contractual agreement and complies with the NACE material sour service guidelines (NACE, 2005). The measured chemical composition was consistent with that for Inconel alloy C276.

\section{Assessment of the functionality of the designed autoclave}

The vessel functionality was tested by carrying out a corrosion test on mini pipe subjected to a combined internal pressure, axial load and elevated temperature. It was shown that the design and set up of autoclaves, in addition to the test protocol, have a dramatic effect on data reproducibility and elimination of errors (McNamee and Conrad, 2011). The vessel structure was secured in the load cell of the testing machine, Instron 4483 by positioning the vessel such that all the fixtures are aligned. The fixtures and mini pipe specimen were then assembled in situ. The mini pipe had an outer diameter of $8 \mathrm{~mm}$ and a wall thickness of $0.25 \mathrm{~mm}$. Test solution was added and electrodes placed in the vessel at appropriate locations and vessel covered with plastic balls to minimise heat loss (top end cap was not used at this initial verification stage), see Figure 11 . The test solution used was $3.5 \mathrm{wt} \% \mathrm{NaCl}$ and $0.1 \mathrm{wt} \%$ with 100 ppmw ammonium bisulphite (ABS); ABS is widely used in oil and gas production wells as oxygen scavenger. After the required test temperature was achieved $\left(90^{\circ} \mathrm{C}\right)$, a designated axial load and internal pressure (by the hydraulic pump) was applied and potentiodynamic measurements were taken using the potentiostat after load stabilisation. 
The vessel was successfully used for corrosion experiment for a mini pipe subject to elevated temperature and combined internal pressure and axial stress. Corrosion was observed on the external surface of the test sample as indicated by the potential versus current plot (Figure 12) and confirmed by SEM image (Figure 13) but not on the internal surface of the vessel as expected due to its corrosion resistance. Passivation was indicated by either constant current or infinitesimal current increment over the finite potential range. As the current and potential increase a breakdown in the passive film is inevitable unless the pipe is immune to corrosion in the environment. When the potential increases to a point where initiation and growth of pits occur, a breakdown potential was determined. The onset of pitting was determined as the potential at which the recorded current density exceeds $100 \mu \mathrm{A} / \mathrm{cm}^{2}$ and does not fall below this value for 60 seconds. The lower the pitting potential the more susceptible the material is to pitting corrosion. The increase in chloride concentration made the pipe more susceptible to pitting corrosion. An understanding of the initiation of pits in corrosive environment is important for design life assessment because pitting is a precursor to stress corrosion cracking as it provides a detrimental combination of local aggressive solution chemistry and stress concentrating features (Turnbull and Zhou, 2004).

The verification showed that the autoclave was suitable for corrosion studies at elevated temperature and combined internal pressure and axial load. The autoclave can be used as standalone or integrated with any tensile equipment, for example, Instron mechanical testing machines (1185 and 4483). It can be moved from one location to the other. Thus, the implications of this new design are two fold; use of the autoclave to carry out corrosion tests of metallic materials or degradation of polymers at HPHT and create a facility that can be used to further research in material and corrosion testing. For corrosion testing this may involve testing under partial pressure of acid gases in an aqueous environment. 


\section{Conclusions}

1. A detailed design of a HPHT autoclave suitable for corrosion studies has been presented. The suitability of an existing HPHT test facility was considered and a range of solutions including lining, coating and autoclave inside an autoclave approach, were considered to adapt the HPHT chamber functionality issues to the proposed test programme. Due to cost and safety issues none of these possible solutions were adopted.

2. A standalone autoclave offered the best approach for addressing the requirements of the high pressure - high temperature corrosion experiments. The design, material specification, and instrumentation of the autoclave have been presented. Inconel alloy C276 was chosen as vessel material for corrosion resistance. The standalone vessel, which was made from Inconel alloy C276, was designed for corrosion tests at maximum temperature of $220{ }^{\circ} \mathrm{C}$ and 15 MPa pressure. The vessel was successfully proof-tested.

3. The autoclave was successfully used to study the initiation of pitting corrosion in super duplex stainless steel pipes in an aqueous solution under a range of a combine internal pressure, axial load and elevated temperature. The autoclave will enable future research studies of corrosion in a wide range of typical oil and gas production and completions, and the interaction between internal pressure, external pressure, temperature and static and dynamic external loading.

4. The maximum design pressure of $15 \mathrm{MPa}$ is more than sufficient for high pressure corrosion studies in aqueous solution where partial pressure of the dissolved gas is one of the main controlling parameters. However, the design pressure is only suitable for corrosion studies in seawater environment up to $1500 \mathrm{~m}$ of water. 


\section{Acknowledgements}

The authors gratefully acknowledge the contribution of Steve Cawley of John Cardwell Limited and Jim Herrmann of Cortest for the manufacture of the autoclave and for the permission to use the vessel design schematic drawings (Figures 8 and 9) in the paper; these figures are not to be used for production without the express written permission of Cortest Inc. The assistance of the technical staff of the School of Engineering Central Workshop is much appreciated.

[1] ReferencesASME Committee. (2007), ASME Boiler and Pressure Vessel Code, Section VIII. Division 1. ASME International.

[2] ASTM G150 (2004), Standard Test Method for Electrochemical Critical Pitting Temperature Testing of Stainless Steel. ASTM.

[3] Bahadori, M. N. (1976), “Design of a solar autoclave”, Solar Energy, Vol. 18, pp. 489-496.

[4] Francis, P. E. and Carter, C. W. (1976), "A pressure vessel for corrosion testing at high temperatures", Journal of Physics E: Scientific Instruments, Vol. 9 No.12, pp.1067-1069.

[5] Holliday, R. I. and Honey, D.J. (1993), “The design of an autoclave for studying hydrometallurgical reactions", Measurement Science and Technology, Vol. 4 No. 9, pp.947-951.

[6] Jenner, G. (1986), “Design of autoclaves”, Physica, Vol. 139 \& 140B, pp. 796 798.

[7] McNamee, K and Conrad, P. (2011), "The effect of autoclave design and test protocol on hydrate test results", Proceedings of the $7^{\text {th }}$ International Conference on Gas Hydrates (ICGH 2011), Edinburgh, Scotland, United Kingdom, July 17-21, 2011

[8] NACE MR0175/ISO 15156. (2005), Petroleum and Natural Gas Industries Materials for use in $\mathrm{H}_{2} \mathrm{~S}$-containing Environments in Oil and Gas Production. NACE/ISO. 
[9] NACE Standard TM0177-96. (1996), Laboratory Testing of Metals for Resistance to Specific Forms of Environmental Cracking in $\mathrm{H}_{2} \mathrm{~S}$ Environments. NACE.

[10] Nice, P., Kopliku, A. and Scoppio, L. (1997), "Materials Selection for Asgard Field Well Tubulars" Paper No. 59, Presented at Corrosion 97, New Orleans, Louisiana.

[11] Oyawale, F. A. and Olaoye, A. E. (2007), "Design and construction of an autoclave", The Pacific Journal of Science and Technology, Vol. 4 No. 2, pp. 224 230.

[12] Turnbull, A., and Zhou, S. (2004), "Pit to crack transition in stress corrosion cracking of a steam turbine disc steel", Corrosion Science, Vol. 46, No. 5, pp.1239-1264

[13] Upadhya, A. R., Dayananda, G. N., Kamalakannan, G. M., Setty, J. R. and Daniel, J. C. (2011), "Autoclaves for aerospace applications: Issues and challenges", International Journal of Aerospace Engineering, Vol. 2011, Article number 985871, doi:10.1155/2011/985871. 


\section{Figure Captions}

Figure $1 \quad$ Existing HPHT test facility

Figure 2 The dimensions of the chamber of exiting HPHT test facility.

All dimensions in millimetres.

Figure 3 Test specimens to be used in the autoclave. (a) C ring specimen. Here A and $B$ are the radius and wall thickness of the pipe from which the ring has been machined. (b) Four-point bend specimen dimensions and fixtures. (c) Uniaxial tensile specimen. (d) Mini pipe specimen.

Figure 4 Specimen and test fixtures assembly inside existing HPHT chamber.

Figure $5 \quad$ Mini pipe fixtures design.

Figure 6 Bellows, with fittings, to be used inside existing HPHT chamber.

All dimensions in millimetres. Courtesy of John Cardwell Limited.

Figure 7 Top and bottom end cap design and seal arrangement for chosen autoclave. All dimensions in millimetres.

Figure 8 Vessel assembly. Courtesy of Cortest Inc.

Figure 9 Cable assembly (thermocouple, pressure transducer, rupture disc etc.) and ports (drain, gas and pressure transducer tubing) assembly. Courtesy of Cortest Inc.

Figure $10 \quad$ Vessel and support infrastructure.

Figure 11 Set up for the verification of the vessel's functionality.

Figure 12 Polarisation scans of $25 \mathrm{Cr}$ super duplex stainless steel mini pipes exposed to $0.1 \mathrm{wt} \%$ and $3.5 \mathrm{wt} \% \mathrm{NaCl}$ with 100 ppmw ammonium bisulphite (ABS) at $90{ }^{\circ} \mathrm{C}$ and subject to a combined internal pressure of 48.3 $\mathrm{MPa}$ and axial tension of $568 \mathrm{MPa}$ 
Figure 13 Micrograph of typical pits on the surface of the tested pipe. 


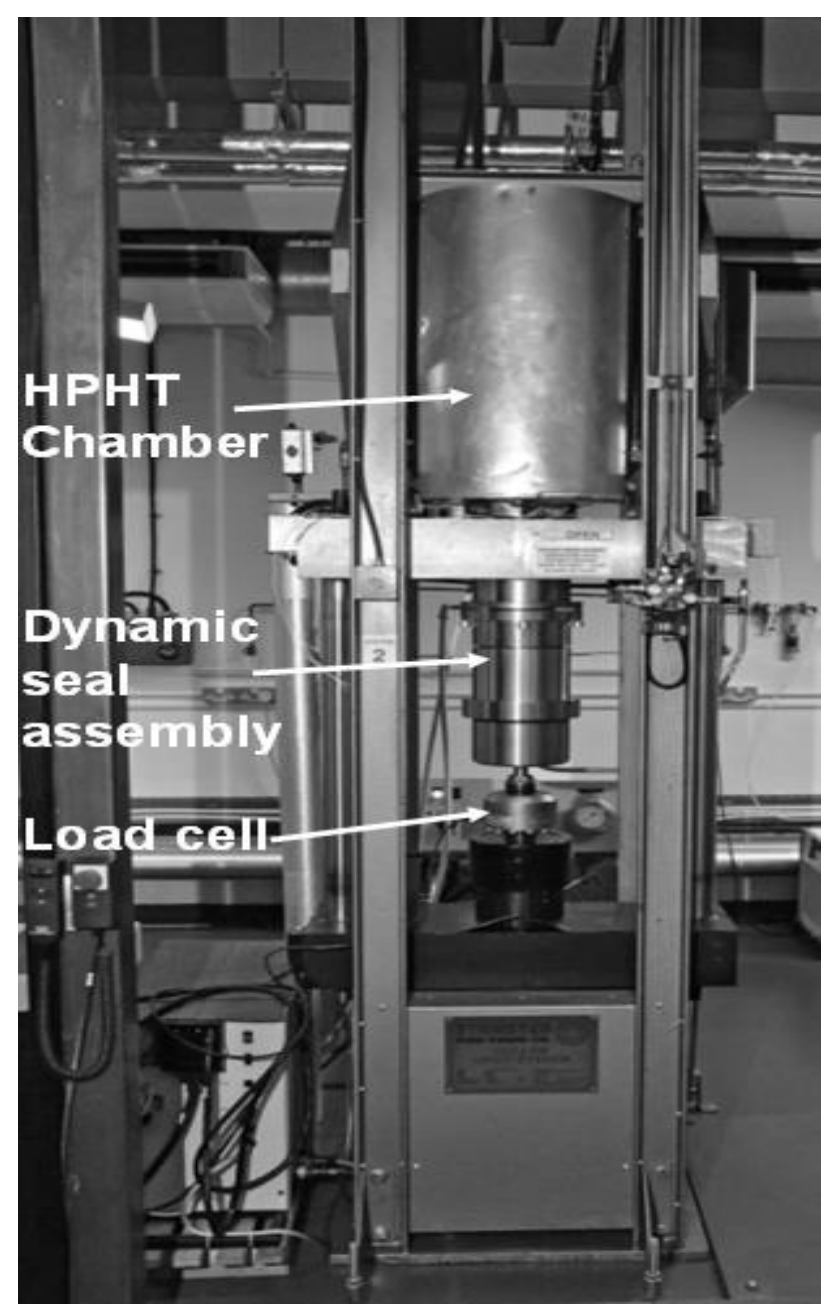

Figure 1: Existing HPHT test facility 


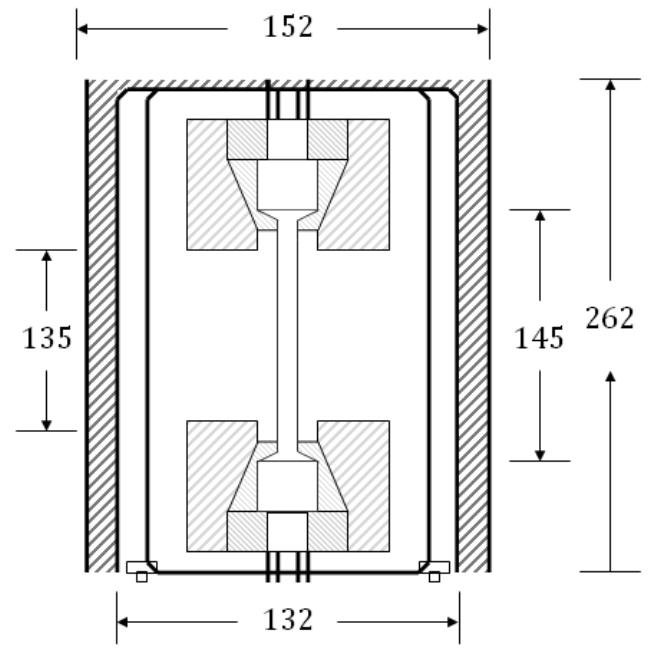

Figure 2: The dimensions of the chamber of exiting HPHT test facility. All dimensions in millimetres. 

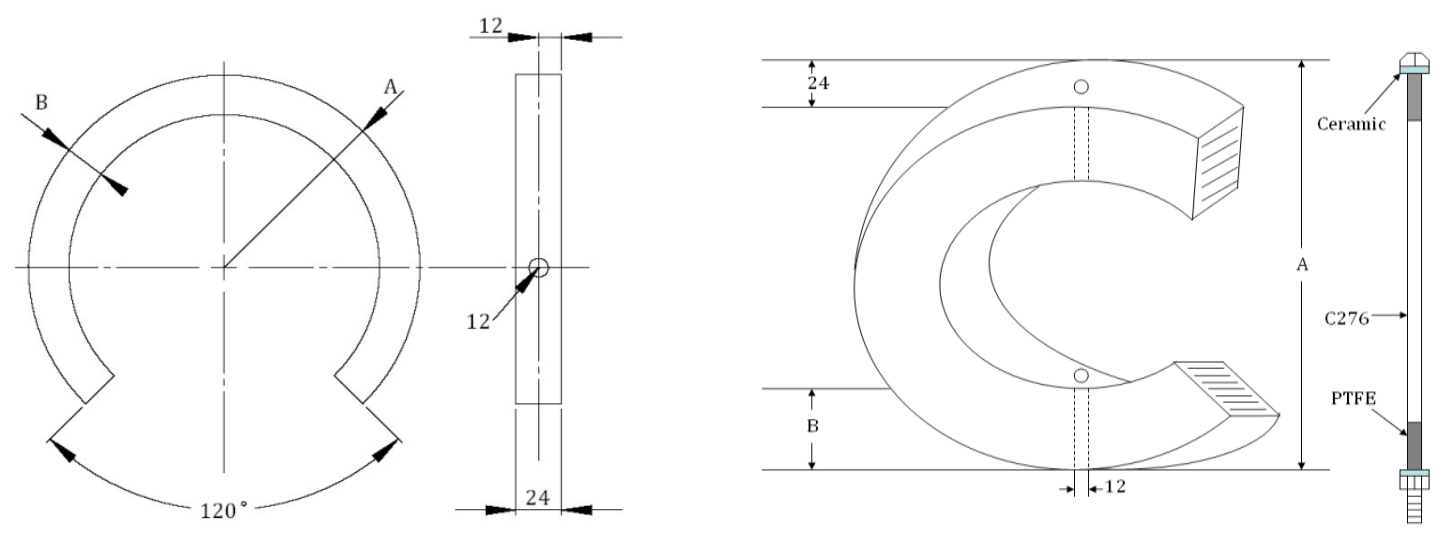

(a)
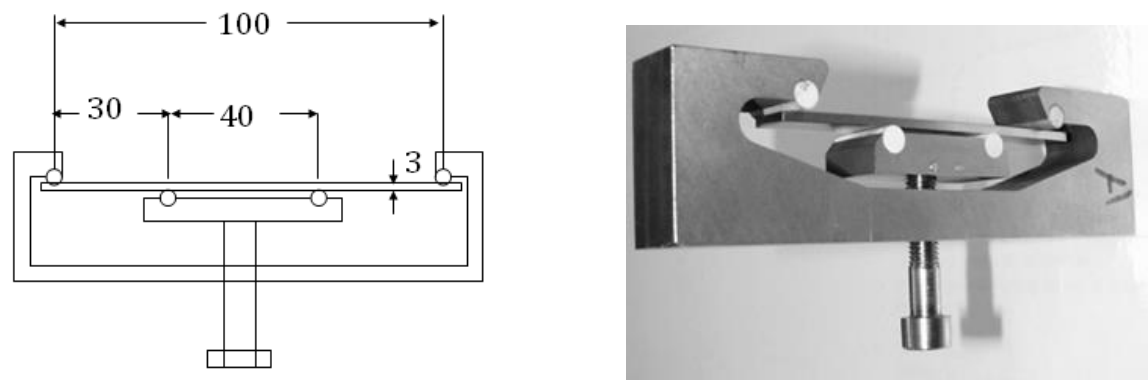

(b)

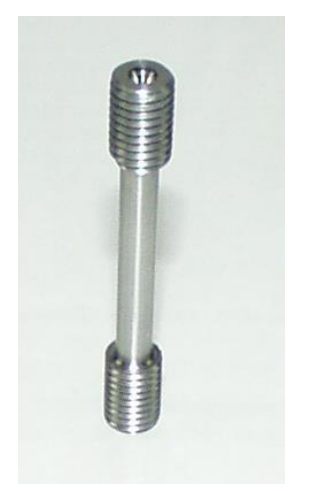

(c)

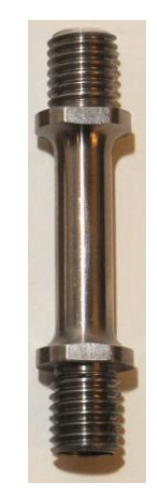

(d)

Figure 3: $\quad$ Test specimens to be used in the autoclave. (a) $\mathrm{C}$ ring specimen. Here A and $B$ are the radius and wall thickness of the pipe from which the ring has been machined. (b) Four-point bend specimen dimensions and fixtures. (c) Uniaxial tensile specimen. (d) Mini pipe specimen. 


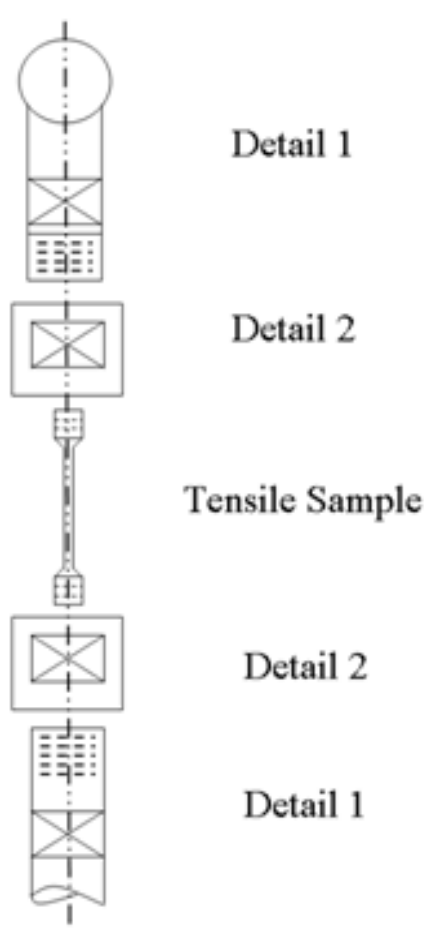

(a) Tensile set-up
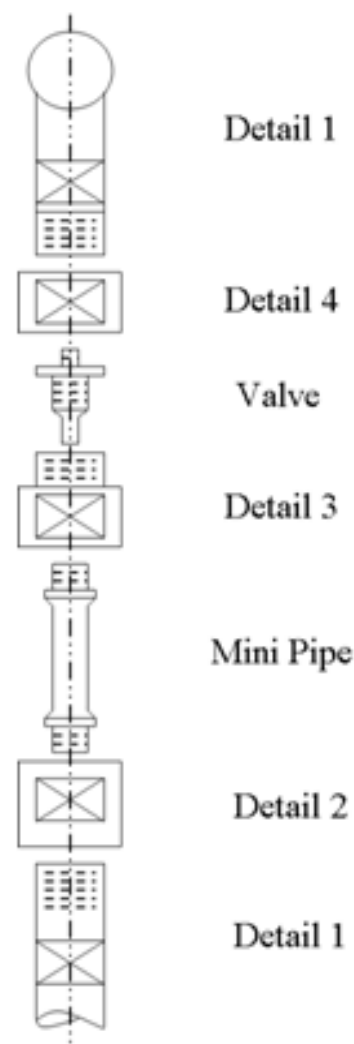

(b) Mini pipe set-up

Figure 4: Specimen and test fixtures assembly inside existing HPHT chamber. 


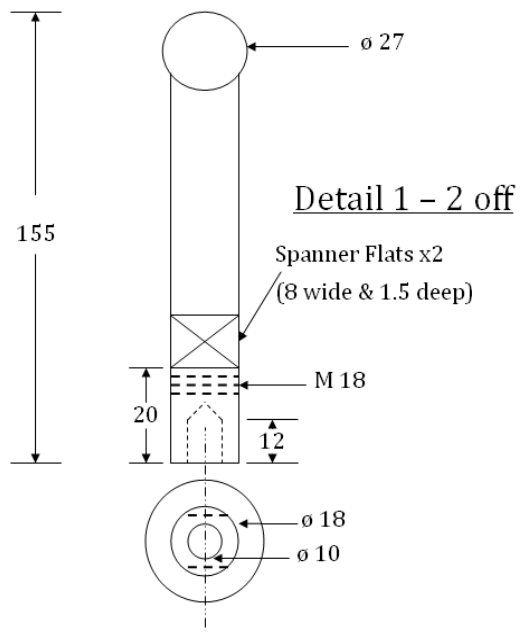

(a)

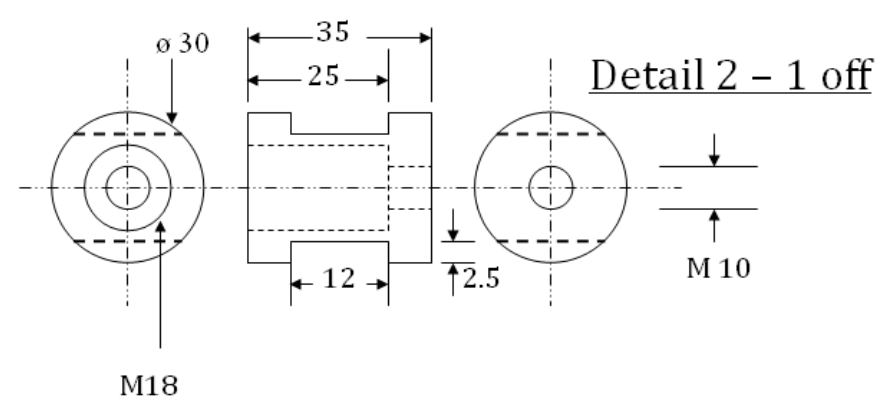

(b)

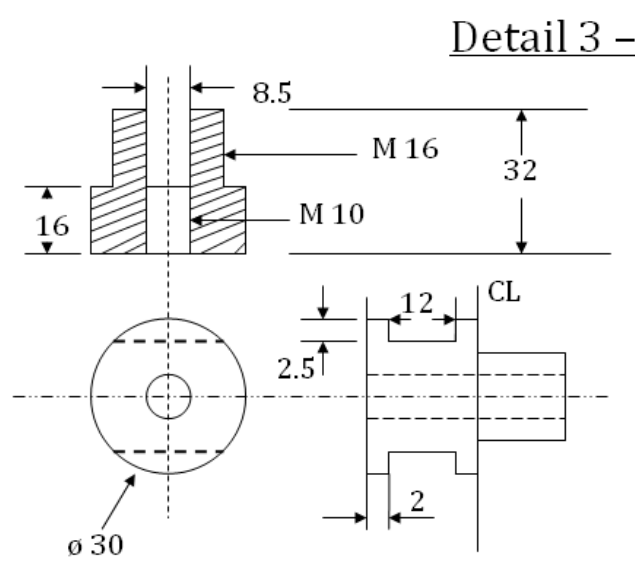

(c)
Detail 4-1 off

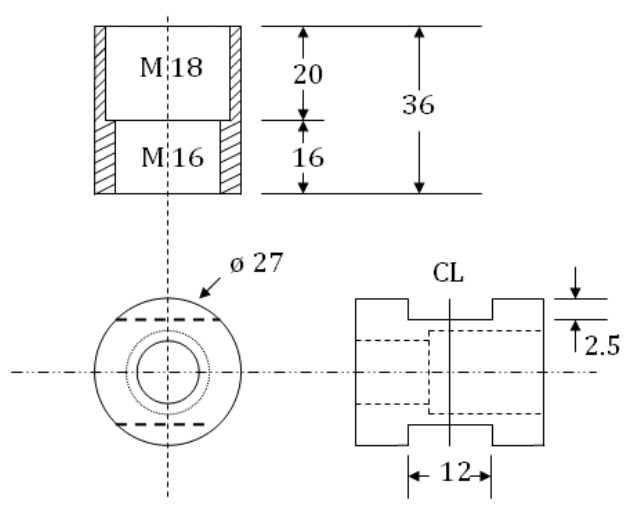

(d)

Figure 5 : Mini pipe fixtures design. 


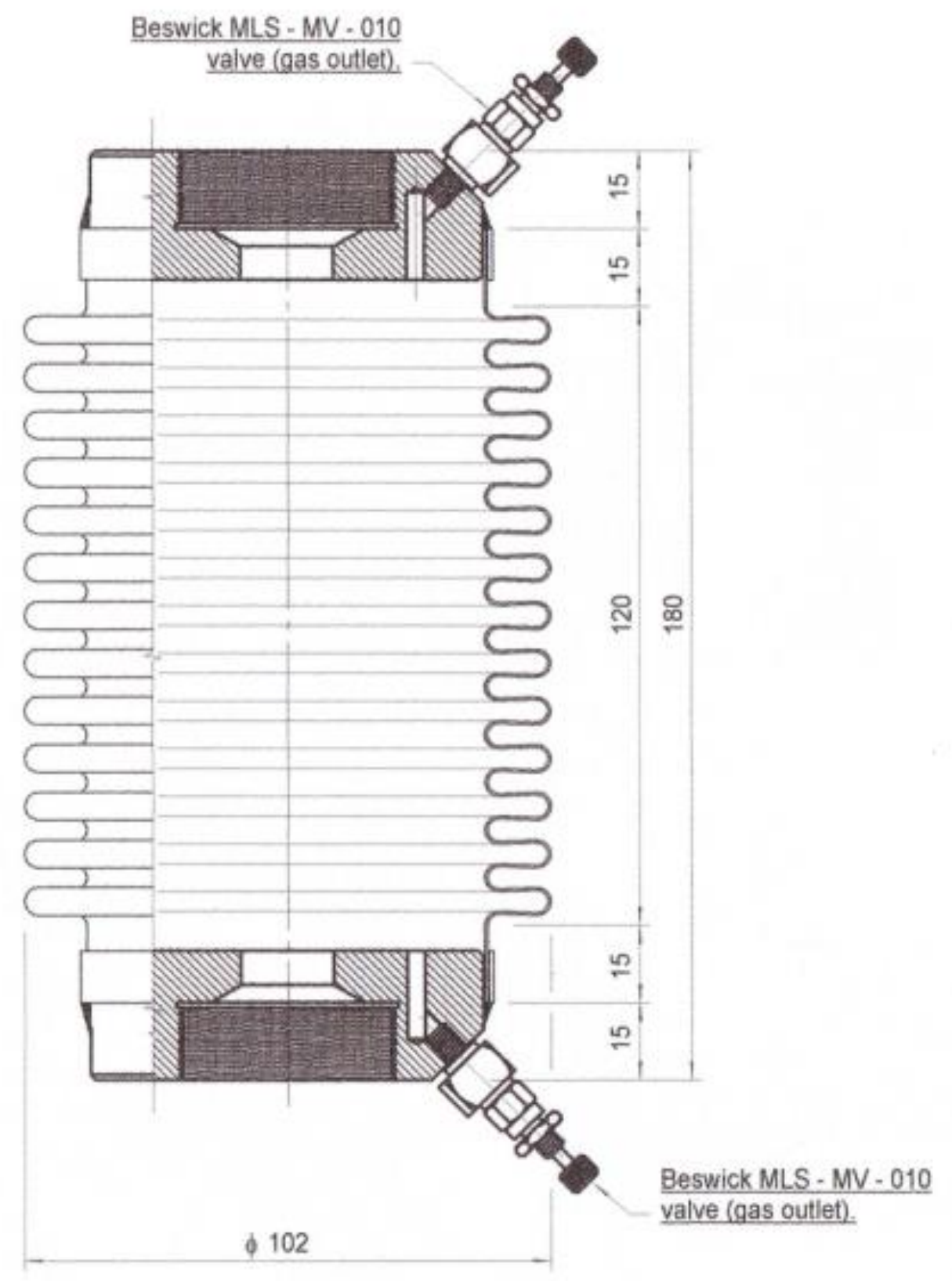

Figure 6: Bellows, with fittings, to be used inside existing HPHT chamber. All dimensions in millimetres. Courtesy of John Cardwell Limited. 


\section{Detail 1 Plate}

8 (12?) holes drilled thro clearance and $\mathrm{C} /$ bored to suit $6(8,10 ?) \mathrm{mm}$ cap screws on 110 P. C. D

\section{Figu}

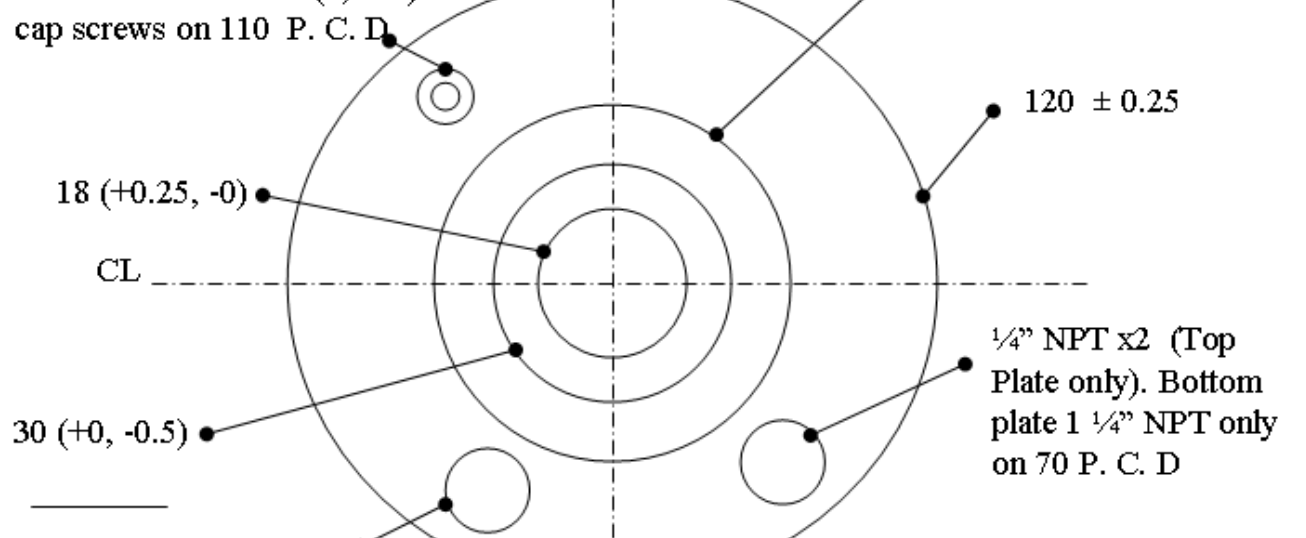

2/3" NPT x2 (Top Plate only) on 70 P. C. D
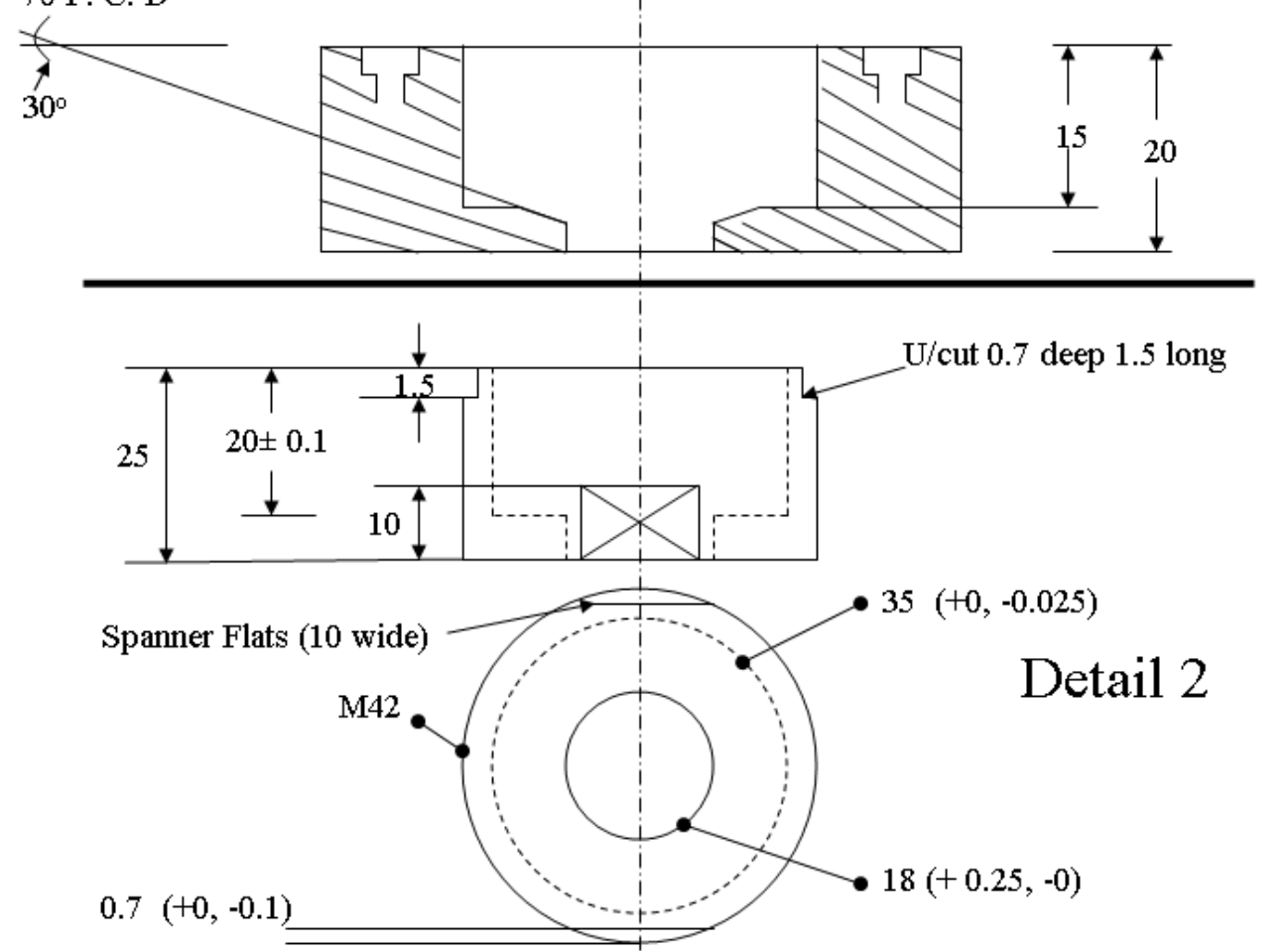


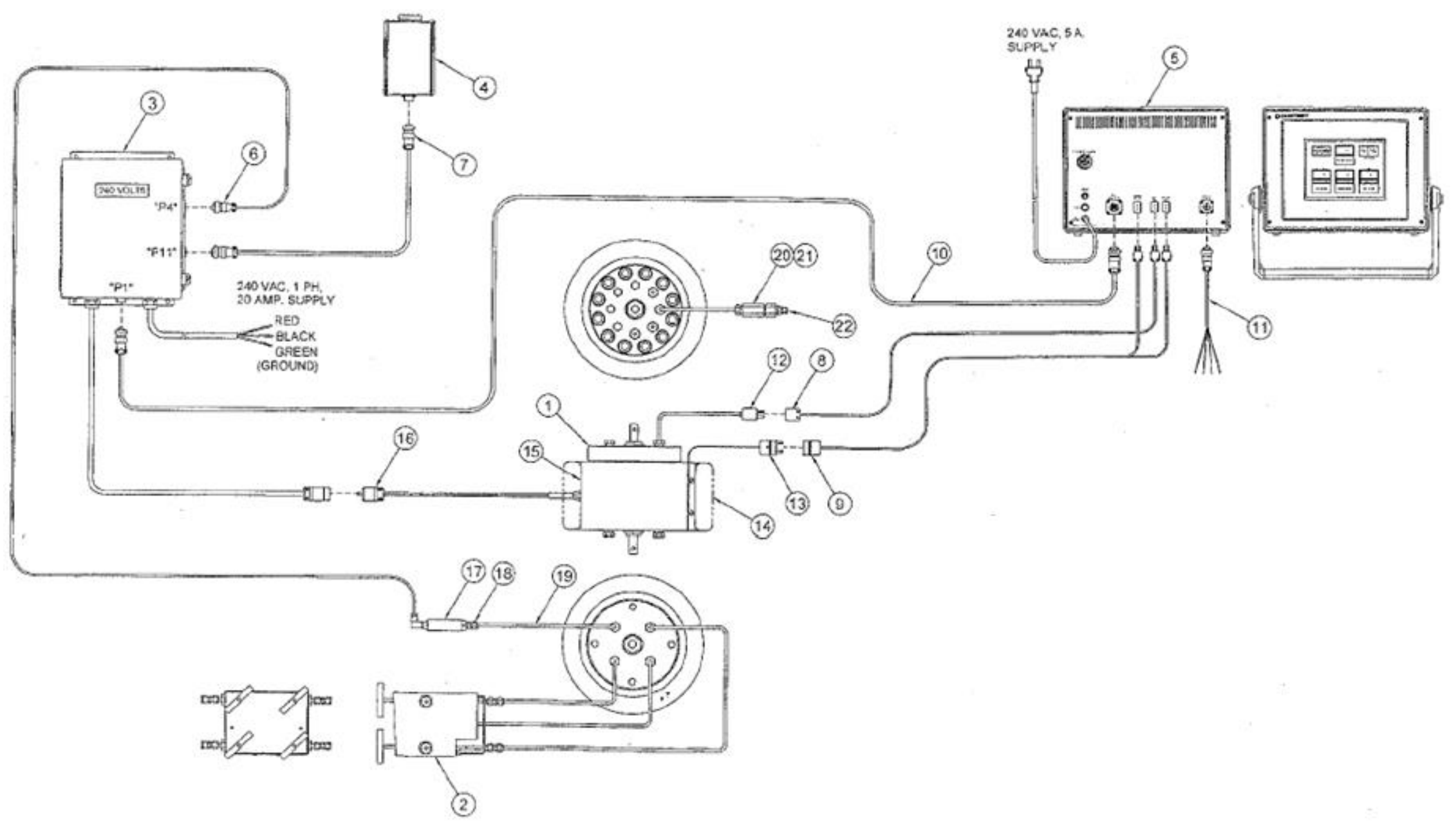

Figure 9 - Caption on next page 


\begin{tabular}{|l|l|}
\hline 22 & Fitting Male Connector \\
\hline 21 & Rupture Disc \\
\hline 20 & Rupture Disc Housing \\
\hline 19 & Tubing \\
\hline 18 & Fitting Male Connector \\
\hline 17 & Pressure Trans ducer \\
\hline 16 & Plug \\
\hline 15 & Heater Band \\
\hline 14 & Insulating Gasket \\
\hline 13 & Thermocouple \\
\hline 12 & Thermocouple \\
\hline 11 & Cable Assembly Analog Output \\
\hline 10 & Cable Assembly SCR Enclosure to AC \\
\hline 9 & Cable Assembly Thermocouple \\
\hline 8 & Cable Assembly Thermocouple \\
\hline 7 & Cable Assembly Digital Pressure Readout \\
\hline 6 & Cable Assembly Transducer \\
\hline 5 & Instrument Assembly \\
\hline 4 & Instrument Assembly Digital Pressure Readout \\
\hline 3 & Control Assembly, SCR \\
\hline 2 & Valve Panel Assembly \\
\hline 1 & Vessel Assembly \\
\hline Item & Description \\
\hline & List of Materials \\
\hline
\end{tabular}

Figure 9: Cable assembly (thermocouple, pressure transducer, rupture disc etc.) and ports (drain, gas and pressure transducer tubing) assembly. Courtesy of Cortest Inc. 


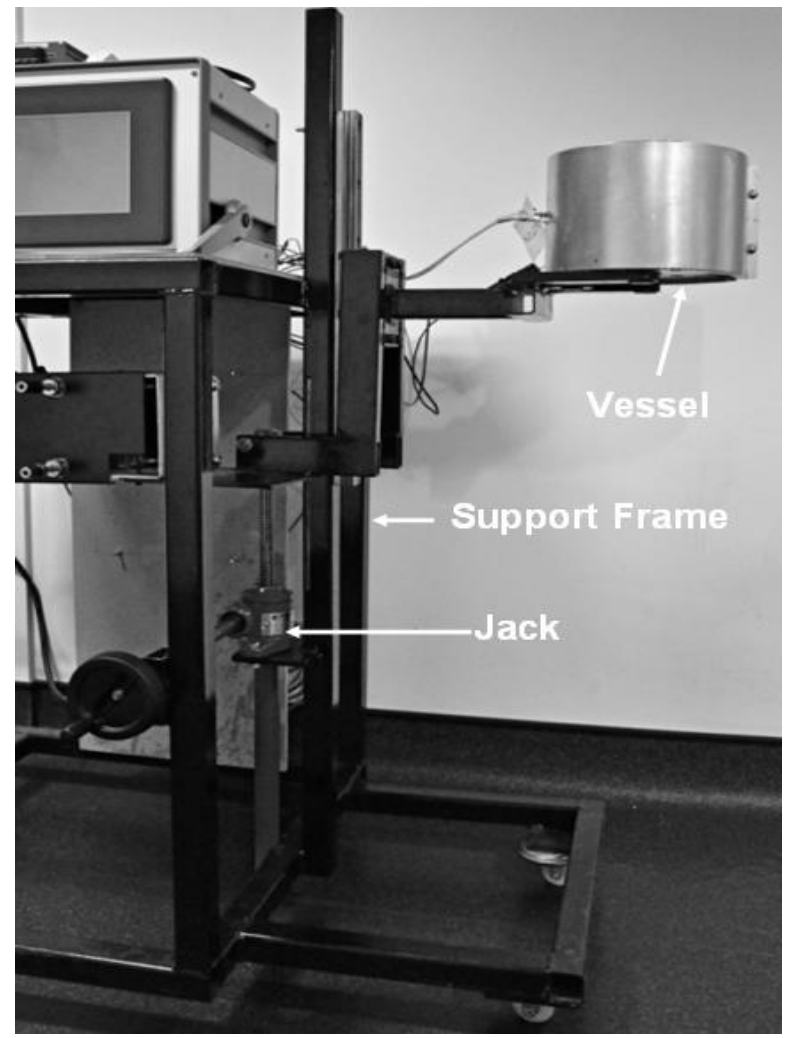

Figure 10: Vessel and support infrastructure. 


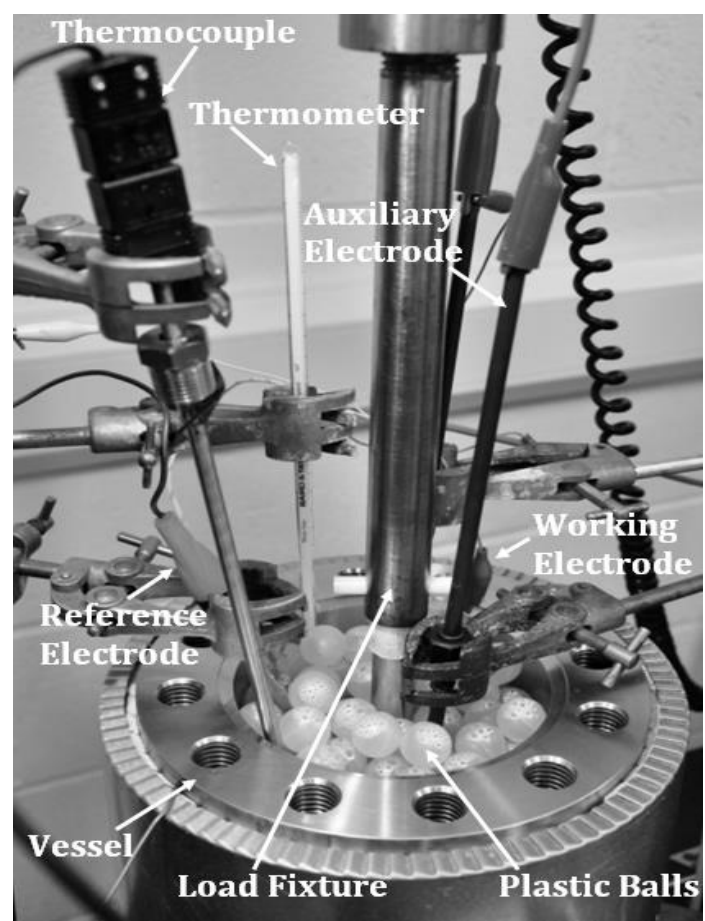

Figure 11: Set up for the verification of the vessel's functionality. 


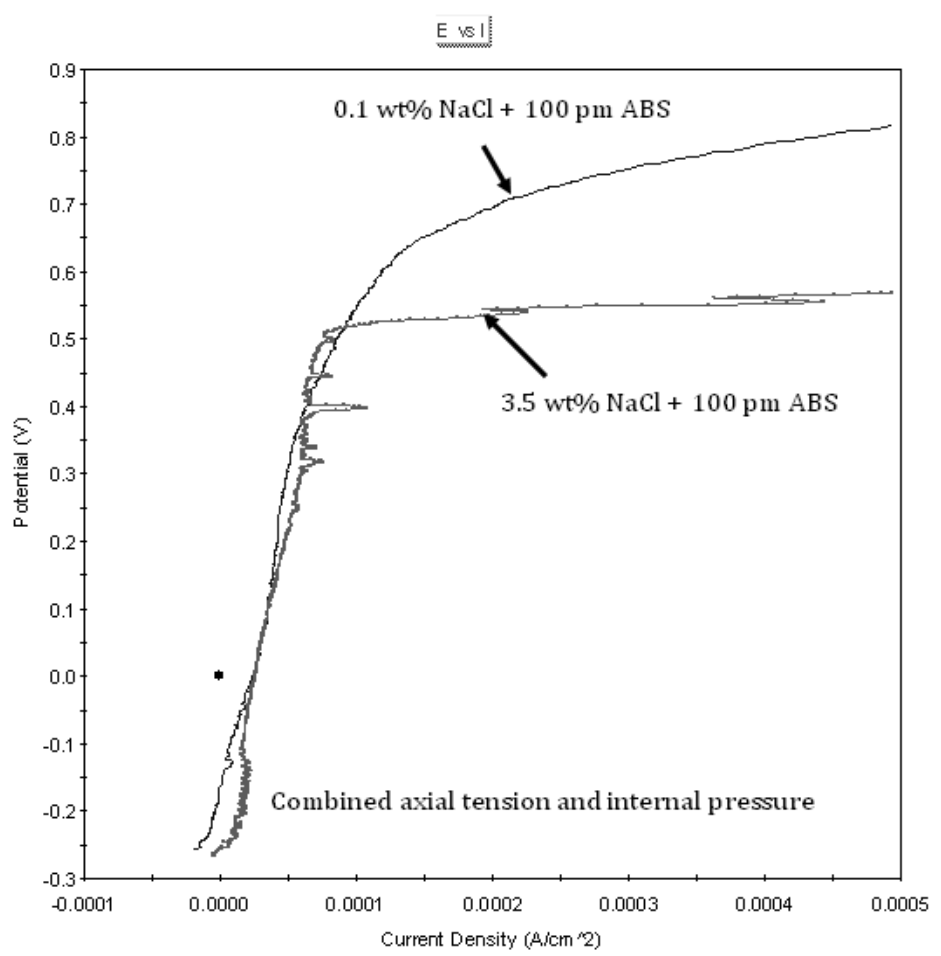

Figure 12: Polarisation scans of $25 \mathrm{Cr}$ super duplex stainless steel mini pipes exposed to $0.1 \mathrm{wt} \%$ and $3.5 \mathrm{wt} \% \mathrm{NaCl}$ with $100 \mathrm{ppmw}$ ammonium bisulphite (ABS) at $90{ }^{\circ} \mathrm{C}$ and subject to a combined internal pressure of 48.3 MPa and axial tension of $568 \mathrm{MPa}$ 


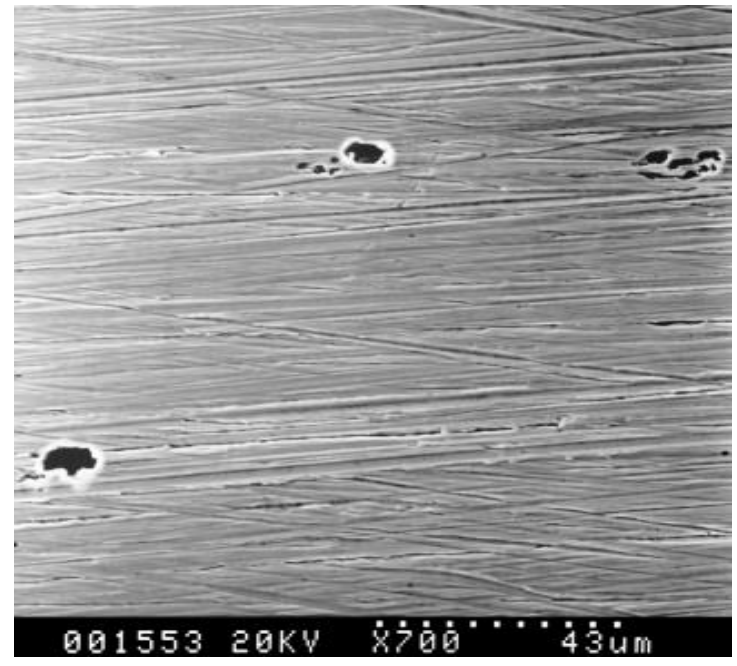

Figure 13: Micrograph of typical pits on the surface of the tested pipe. 